\title{
Role of MRI special diffusion weighted imaging/diffusion tensor imaging techniques in the assessment of perianal fistula activity
}

\author{
Radwa ElSharbatly", Manal Halim, Fady Shaker, Abdrabou N. Mashhour and Heba Azzam
}

\begin{abstract}
Background: Pre-operative MRI examination is currently considered as an evolving tool for assessment of perianal fistula types, extensions, and complications to achieve proper treatment plan. Proper assessment of fistula activity can also have a major contributing role to the treatment plan, through deciding the type of prescribed medications, proper surgical approach as well as the proper operative intervention timing. Reviewing literature, only few studies have mentioned the importance of diffusion tensor imaging (DTI) sequences in diagnosing perianal fistula activity, yet many studies have discussed the perianal fistula activity assessment using other diffusion weighted imaging (DWI) sequences. In the present study, the main objective was to prove the MRI quantitative DTI sequences' ability of diagnosing perianal fistulae inflammatory activity.

Results: This study was a prospective analysis in which fistular activity was confirmed by intra-operative findings (considered as the standard reference). The cases included in the study were divided into two groups, based on their surgical findings, positive inflammatory and negative inflammatory groups. Both groups were pre-operatively assessed using MRI imaging and additionally used diffusion weighted and tensor imaging (DWI and DTI) sequences by special post-processing quantitative assessment of DTI FA and ADC values. There was significant statistical difference between the mean ADC value of the PIA and NIA groups in the track, edema, ipsilateral and contralateral sphincter areas with $P$ values ( $P=0.000,0.000,0.002$ and 0.000 respectively). There was also significant difference between the mean FA value of the PIA and NIA groups in the track, edema, ipsilateral and contralateral sphincter areas with $P$ values ( $P=0.000,0.000,0.000$ and 0.008 respectively).

Conclusion: This study results revealed that FA are relatively lower in positive inflammatory activity lesions than in negative inflammatory activity lesions while the ADC values were relatively higher in positive inflammatory activity lesions than in negative inflammatory activity lesions and the differences were statistically significant having a fundamental role in the assessment of perianal fistula activity especially at the track area being of highest sensitivity and specificity. Unlike conventional MRI sequences which revealed only high specificity being a good negative modality.
\end{abstract}

Keywords: Diffusion weighted imaging, Diffusion tensor imaging, DTI, ADC value, FA value, Perianal fistula activity, Magnetic resonance imaging

*Correspondence: r.s.elsharbatly@kasralainy.edu.eg

Kasr Alainy School of Medicine, Cairo University, Kasr Alainy St, Cairo, Egypt

\section{Background}

Perianal fistula is an inflammatory process that primarily affects the anal canal and its surroundings, it can cause significant morbidity and, in many cases, could require multiple operative interventions due to its high incidence of recurrence [1]. However, magnetic resonance (MR) imaging can help more precise anatomical identification 
of the fistula and its relations to the anal canal, the anal sphincter complex, and to the pelvic floor structures and the plane of the levator ani muscle, this also helps detection of secondary fistulas or abscesses [1]. Proper fistular activity assessment can be of major role contributing to the treatment plan, by choosing the number of administered drugs, deciding the proper surgical approach and also choosing the proper operative intervention timing [2].

Reviewing literature, only few studies have mentioned the importance of diffusion-weighted imaging (DWI) and diffusion tensor imaging (DTI) sequences in diagnosing anal fistulas and perianal abscesses [3] yet many studies have discussed the perianal fistular activity [4].

In the current study we aimed to evaluate the feasibility of detection of perianal fistula activity by analysis and quantitative assessment of the DTI (FA and ADC) values.

\section{Methodology}

This study was a prospective analysis study, approved by the ethics committee at our institute and was conducted during the period from December 2018 to December 2020.

Fistular activity was confirmed by intra-operative findings (considered as the standard reference) by differentiating the cases into two groups (positive and negative inflammatory groups) based on the presence of necrotic tissues, pus and other inflammatory products.

\section{Patient characteristics}

Sixty-two patients were included in this study (Fig. 1), fifty-five (88.7\%) were males and seven (11.3\%) were females with age ranging from twenty-three to sixty-nine with mean \pm SD of $38.74 \pm 10.51$. Their age ranged from 23 to 69 years (mean age $38.74 \pm 10.51$ ).

The forementioned patients were referred to the radiology department by surgery outpatient clinic requesting preoperative MRI assessment. Based on the surgery conclusions and clinical evaluation (performed by two surgeons of 4 years -resident- and 8-10 years' experience -senior registrar/consultant-).

Different types of fistulae were diagnosed, thirty-three of the cases were of inter-sphincteric type (fifteen active and 18 inactive) seventeen were of the trans-sphincteric type (twelve active and 5 inactive) and twelve cases were of the extra-sphincteric type (five active and 7 inactive).

All the referred patients were operated upon by senior surgeon (consultant) with 8-10 years' experience-who were blinded as regards the MRI DWI/DTI findings - and stated in their feedback that patients with

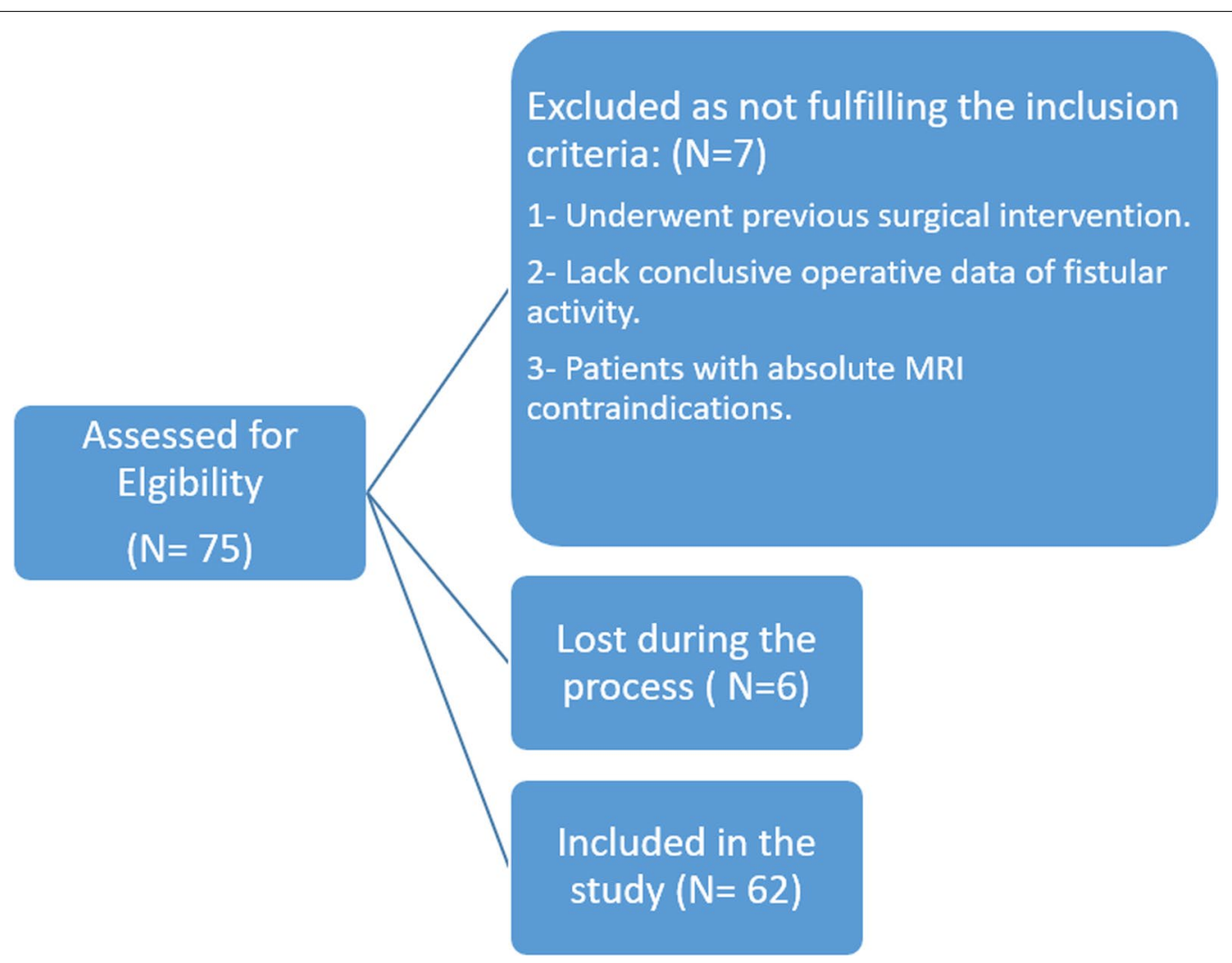

Fig. 1 Flow chart of included patients 


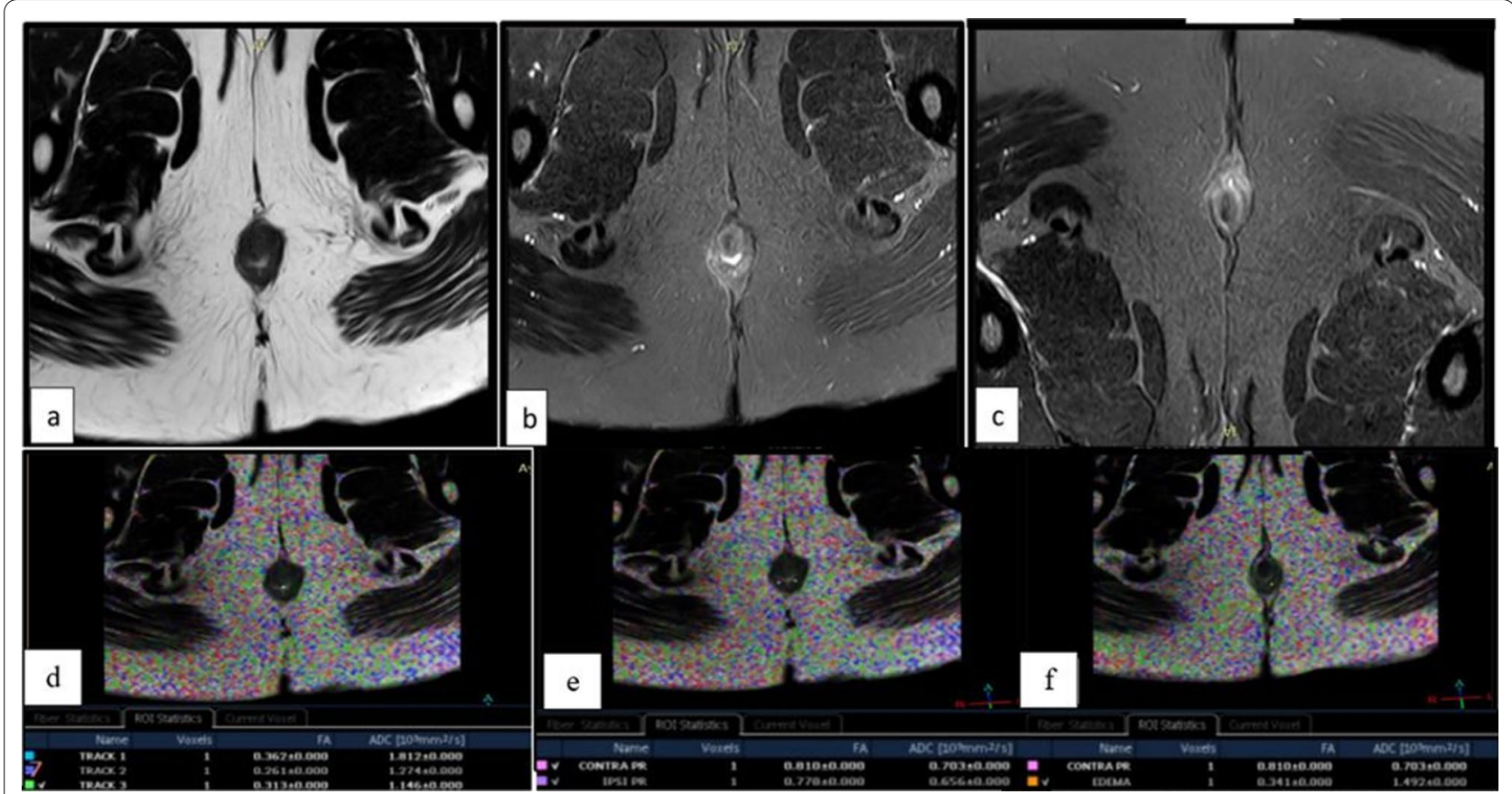

Fig. 2 a axial T2 WI, b axial STIR WI, c coronal STIR WI for an inter-sphincteric track of bright signal in T2 and STIR WI extending from 4 to 8 O'clock surrounding the internal sphincter. axial DTI/T2 fused images with ROI (FA and ADC) values placed in the inter-sphincteric track (d), ipsilateral and contralateral sphincters (e) and surrounding edema (f) with FA values $0.313,0.778,0.810$ and 0.341 and ADC values $1.146,0.656$ and $0.703,1.492 \times$ $10^{3} \mathrm{~mm}^{2} / \mathrm{s}$, respectively. The FA and ADC values are relatively low indicating active inflammatory condition

inter-sphincteric fistulae were operated upon by simple fistulotomy (Lay open) technique while trans-sphincteric and extra-sphincteric cases were operated upon by sphincter preserving techniques mainly fistulectomy (coring) and repair of the sphincter if needed.

These patients were divided accordingly into two groups: the positive inflammation activity (PIA) group -being thirty-two (51.6\%)-with thirty males and two females- and the negative inflammation activity (NIA) group -being thirty (48.4\%)- with twenty-five males and five females.

\section{Inclusion criteria}

Patients proved to have perianal fistulae that require preoperative assessment.

\section{Exclusion criteria}

These involved patients that underwent previous surgical intervention, patients who lack conclusive operative data of fistular activity, patients with absolute MRI contraindications as having pacemakers or intracranial aneurysmal clips as well as claustrophobic patients.

\section{MR imaging}

MRI was performed using 1.5 Tesla magnet scanner by two devices (Intera and Achieva, Philips medical system). All patients were examined in the supine position using a surface coil, Subjects were required to empty their bladders before the examinations. No sedation was used.

The MRI imaging protocol included the following sequences:

Turbo spin echo (TSE) T1-weighted imaging (T1WI): axial; TR/TE, 600/10 ms; field of view (FOV), $200 \times 200 \mathrm{~mm}^{2}$; matrix, $400 \times 400$; slice thickness, $5 \mathrm{~mm}$; and number of slices, 20. TSE T2-weighted imaging (T2WI): axial, sagittal and coronal; TR/TE, $1560 / 80 \mathrm{~ms}$; FOV, $200 \times 200 \mathrm{~mm}^{2}$; matrix, $400 \times 400$; slice thickness, $5 \mathrm{~mm}$; and number of slices, 20 . Short T1 inversion recovery imaging (STIRWI): axial, sagittal and coronal; 200$300 \mathrm{~ms}$; matrix, $236 \times 147 \mathrm{~mm}^{2}$; slice thickness, $5 \mathrm{~mm}$; and number of slices, 20. DWI and DTI (spin echo-echoplanar imaging, SE-EPI): axial; TR/TE, 3250/48 ms; FOV, $200 \times 200 \mathrm{~mm}^{2}$; matrix, $80 \times 80$ with a $112 \times 112$ reconstructed matrix; slice thickness, $5 \mathrm{~mm}$; number of slices, 20; 32 diffusion-weighed directions; NSA, $1 ; b=0.400 \mathrm{~s} /$ $\mathrm{mm}^{2}$. Other $\mathrm{b}$ values acquisition was done in some cases with $b=400,600$ and $1000 \mathrm{~s} / \mathrm{mm}^{2}$. Respiratory triggering was used for better resolution. 

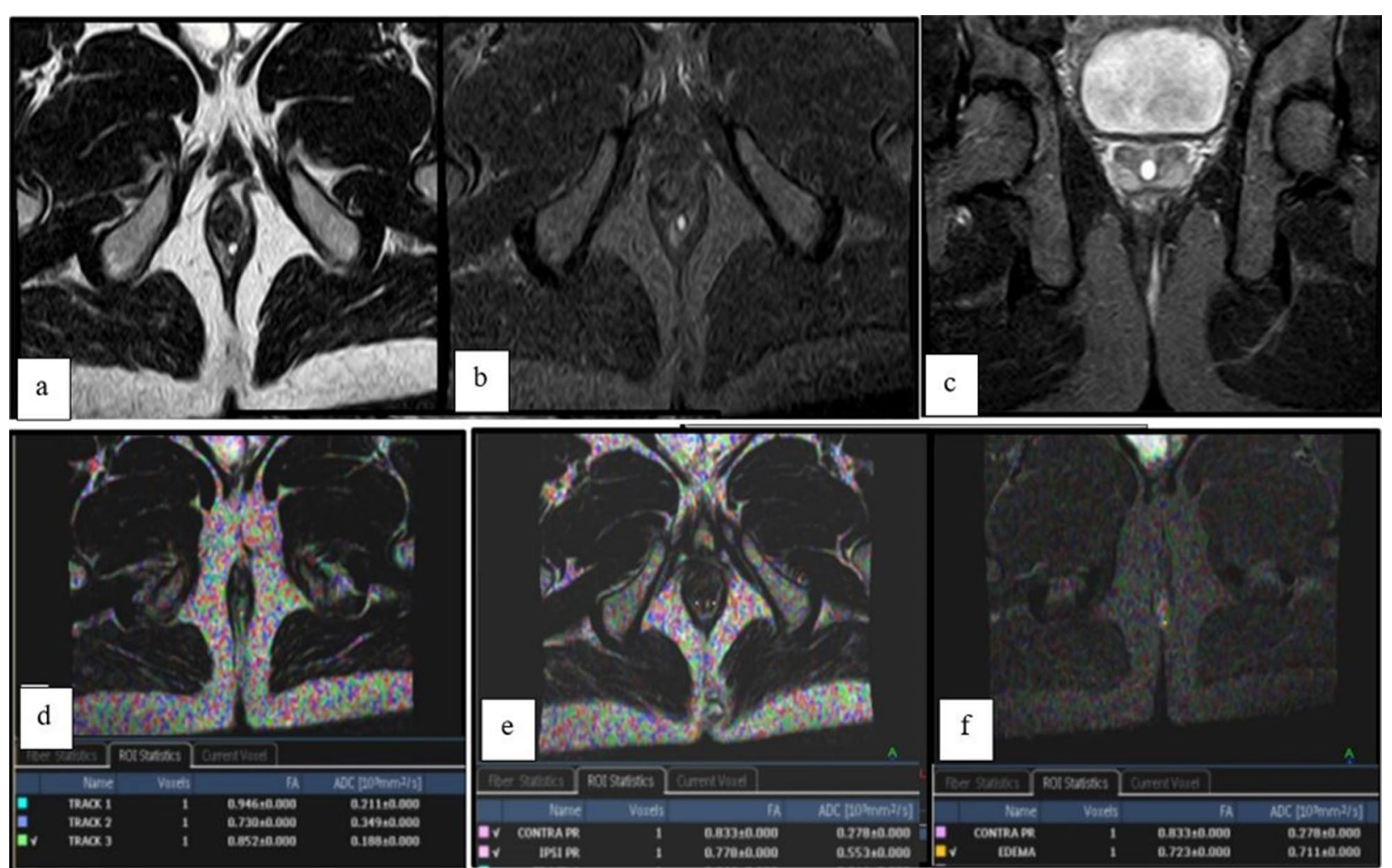

Fig. 3 a axial T2 WI, b STIR WI and c coronal STIR WI for a linear inter-sphincteric track of intermediate/high signal in T2/STIR WI touching the internal sphincter at 4-5 O'clock. Axial DTI/T2 fused images with ROI (FA and ADC) values placed in $\mathbf{d}$ the inter-sphincteric track, e ipsilateral and contralateral sphincters and $\mathbf{f}$ surrounding edema area with FA values $0.852,0.778$, and 0.833 and 0.723 with ADC values $0.188,0.553,0.278$ and $0.711 \times 10^{3} \mathrm{~mm}^{2} / \mathrm{s}$, respectively. The FA and ADC values are relatively high indicating inactive inflammatory condition

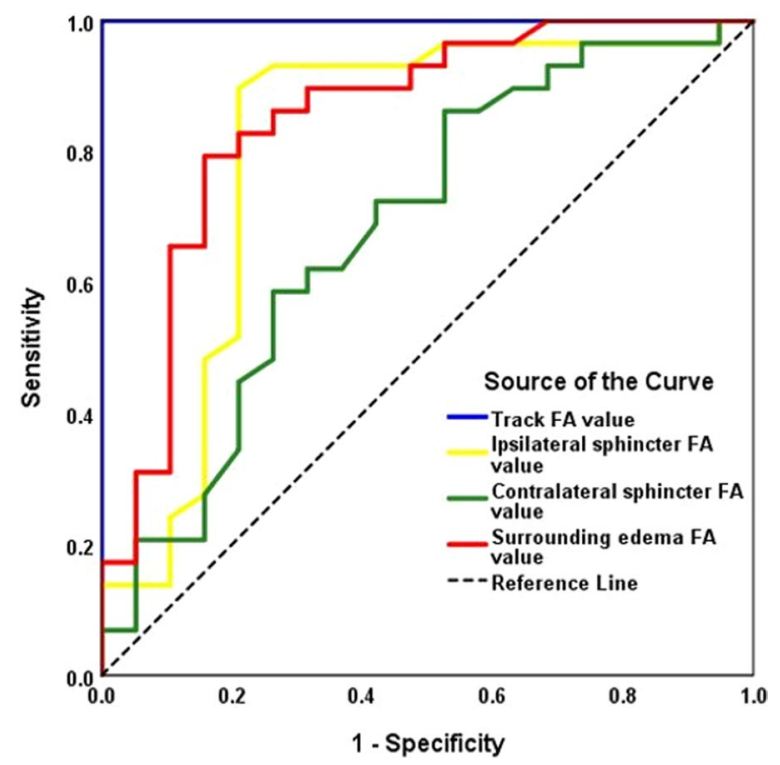

Fig. 4 ROC curve analysis for FA measures to differentiate active conditions
Post processing and image analysis were conducted as follows and qualitative as well as quantitative analysis of the MR images were conducted as a double-blinded analysis by two radiologists one with 5 years (R.E.) experience and the other with 15 years (H.A.) experience.

\section{Qualitative analysis}

Restricted diffusion was determined by visualization of abnormal bright signal intensity that became enhanced with increasing $b$ values at "Diffusion weighted" (DW) images. The ADC map presented intermediate/low signal intensity (SI) that corresponded to the abnormality. The DTI images were fused with T2/STIR WI to allow the assessment of the anatomical details.

\section{Quantitative analysis}

The ADC values were measured manually by applying ROI on the DTI $(b=0)$ fused images on the largest suspected area, and overlaid on FA and ADC maps, and the average value was obtained by measuring 3 times. In addition, the lesions were divided into 4 ROIs (i.e., the fistula area, edema area, ipsilateral affected sphincter 
Table 1 ROC curve analysis for FA measures

\begin{tabular}{lllllcc}
\hline & AUC & $\mathbf{9 5 \% C l}$ & P value & Cut-off & Sensitivity (\%) & Specificity (\%) \\
\hline Track FA value & 1.000 & $1.0-1.0$ & $\mathbf{0 . 0 0 0}$ & $\leq 0.410$ & 100.0 & 100.0 \\
Ipsilateral sphincter FA value & 0.808 & $0.661-0.954$ & $\mathbf{0 . 0 0 0}$ & $\leq 0.522$ & 89.7 & 78.9 \\
Contralateral sphincter FA value & 0.684 & $0.525-0.843$ & $\mathbf{0 . 0 3 2}$ & $\leq 0.717$ & 62.1 & 68.4 \\
Surrounding edema FA value & 0.850 & $0.731-0.969$ & $\mathbf{0 . 0 0 0}$ & $\leq 0.505$ & 79.3 & 84.2 \\
\hline
\end{tabular}

$P$ value is considered statistatically significant when less than 0.005

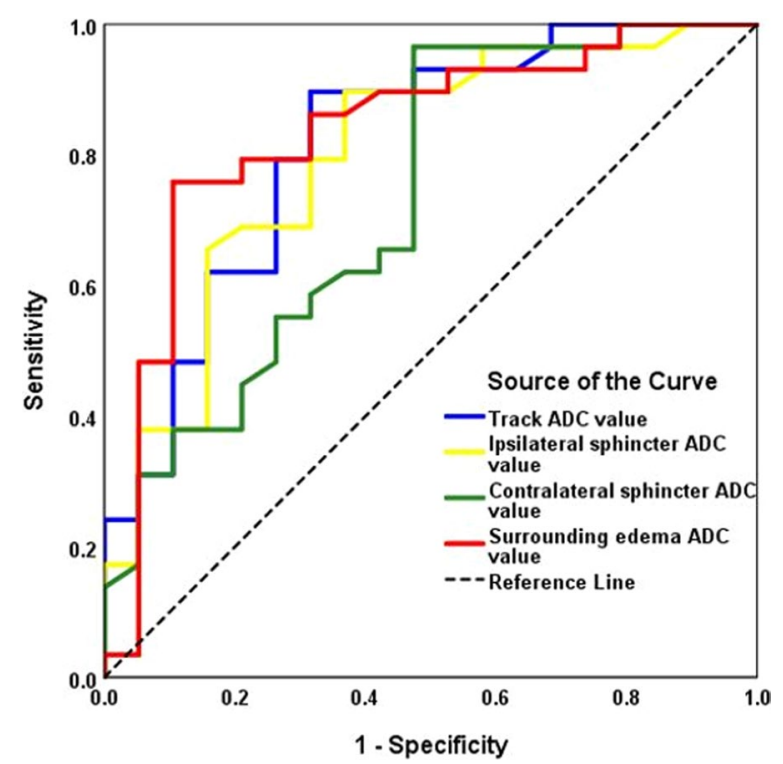

Fig. 5 ROC curve analysis for ADC measures to differentiate active conditions

area and distant normal-appearing contralateral sphincter area).

\section{Statistical analysis}

All statistical analyses were conducted with the Statistical Package for Medical Statistics (Medcalc15.8, Ostend, Belgium; https://www.medcalc.org). All the FA, ADC values are presented with mean \pm standard deviation $(x \pm s)$. The FA and ADC values of each area were calculated and analyzed with single-factor analysis of variance (ANOVA). The DTI values of each group were statistically calculated with independent samples $t$ tests. The cut-off values for the ADC and FA measurements in evaluating the activity of the lesions were obtained by receiver operating characteristic (ROC) curve analysis, as were the sensitivity and specificity. In all tests, $P<0.05$ represented statistically significant differences.

Sample size.

Epi-calc 2000 was used to calculate the sample size of this cross-sectional study. Assuming $80 \%$ power, 0.05 level of significance, $20 \%$ null hypothesis value and estimated proportion of $38 \%$.

Sample size $=43$.

\section{Results}

\section{DTI quantitative analysis}

Analysis of the diffusion tensor images and their correlation with the clinical condition was conducted using double blinded analysis by two radiologists with interobserver variability of 0.6 .

In positive inflammatory activity group (Fig. 2), the DTI assessment revealed relatively lower FA and higher ADC values than in negative inflammatory activity group (Fig. 3), with the FA in the PIA group measured $0.12-0.41,0.11-0.85,0.18-0.93$ and $0.23-0.94$ in the track area, edema area, ipsilateral and contralateral sphincter areas, respectively. While in the NIA group FA measured $0.33-0.94,0.26-0.99,0.22-1$ and $0.39-1$ in the track area, edema area, ipsilateral and contralateral sphincter areas, respectively.

Similar findings were detected regarding the ADC values $\left(\times 10^{-3} \mathrm{~mm}^{2} / \mathrm{s}\right)$ which were higher at the PIA group as the ADC in the track area, edema area, ipsilateral and contralateral sphincter areas measured

Table 2 ROC curve analysis for ADC measures

\begin{tabular}{|c|c|c|c|c|c|c|}
\hline & AUC & $95 \% \mathrm{Cl}$ & $P$ value & Cut-off & Sensitivity (\%) & Specificity (\%) \\
\hline Track ADC value & 0.816 & $0.689-0.942$ & 0.000 & $\geq 0.999$ & 89.7 & 68.4 \\
\hline Ipsilateral sphincter ADC value & 0.799 & $0.668-0.931$ & 0.001 & $\geq 0.578$ & 89.7 & 63.2 \\
\hline Contralateral sphincter ADC value & 0.732 & $0.583-0.882$ & 0.007 & $\geq 0.545$ & 96.6 & 52.6 \\
\hline Surrounding edema ADC value & 0.834 & $0.708-0.960$ & 0.000 & $\geq 0.733$ & 86.2 & 68.4 \\
\hline
\end{tabular}

$P$ value is considered statistatically significant when less than 0.005 


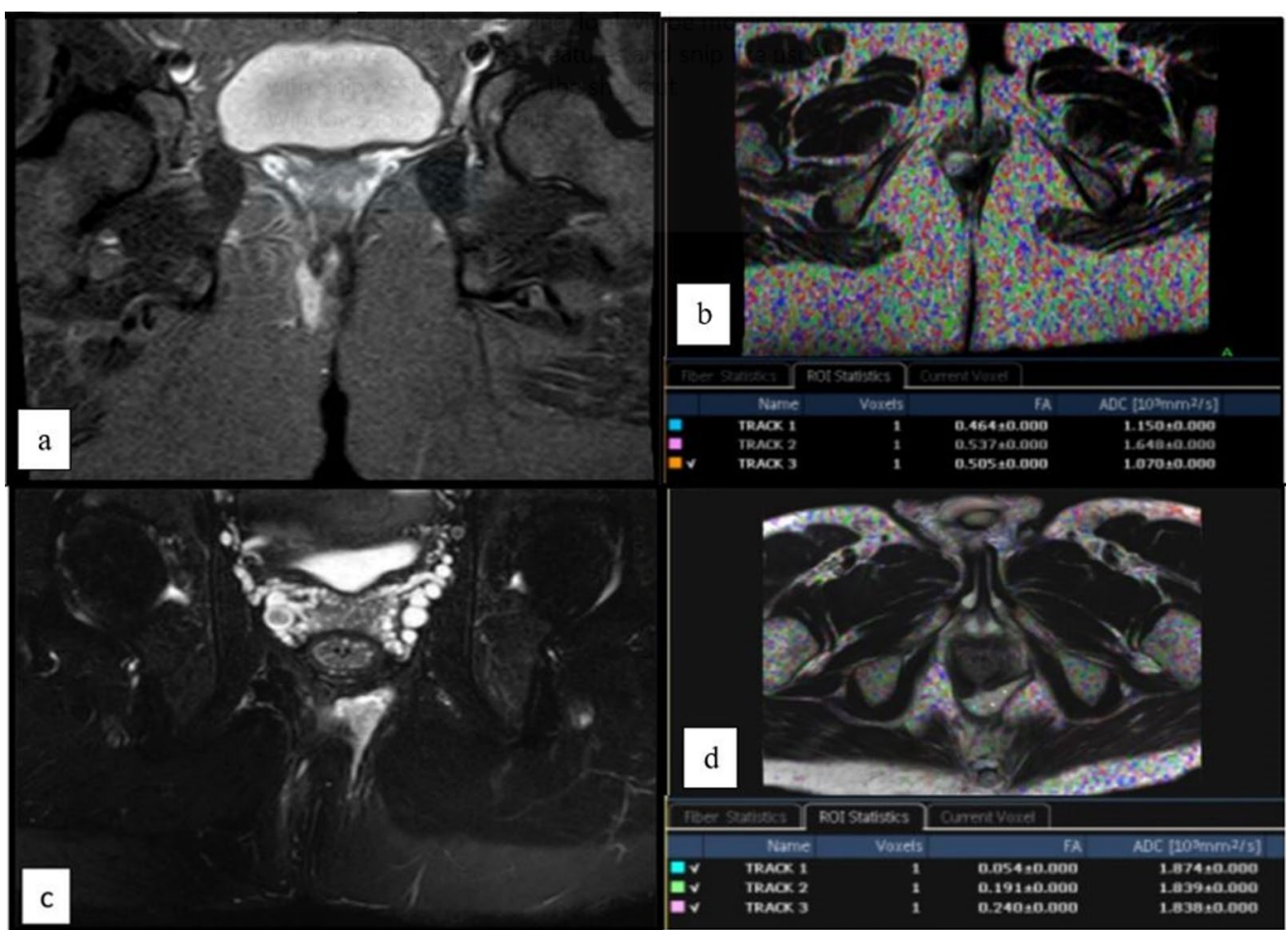

Fig. 6 a Coronal STIR WI, b axial DTI/T2 fused images with ROI (FA and ADC) placed in the trans-sphincteric showing high track FA (0.505) and ADC values (1.070) indicating inactive condition with c coronal STIR WI, d axial DTI/T2 fused images with ROI (FA and ADC) placed in the saddle trans-sphincteric track from 4 to 7 O'clock showing low track FA (0.191) and ADC values (1.839) indicating active condition

$0.5-2.66,0.28-2.19,0.22-1.8$ and $0.19-2.12$, respectively, and in the NIA, it measured $0.1-1.62,0.02-2.05$, $0.03-1.98$ and $0.02-1.73$. respectively.

There were statistically significant differences in the DTI (FA and ADC) values of the fistula area, edema area, ipsilateral and contralateral sphincter areas with $P$ values for FA $0.000,0.000,0.000$ and 0.032 , while for the ADC were $0.000,0.000,0.001$ and 0.007 , respectively.

ROC curve analysis for FA measures (Fig. 4) to differentiate active conditions, considering cut off values for FA (Table 1) showed sensitivity and specificity for the track area reaching $100 \%$, while that for the edema area reaching $79 \%$ and $84 \%$, respectively.

ROC curve analysis for ADC measures (Fig. 5) to differentiate active conditions fixing the cut off values for ADC (Table 2) showed sensitivity for the track and ipsilateral sphincter areas reaching $89.7 \%$, while for the contralateral sphincter and edema areas reaching more than $96 \%$ and $86 \%$, respectively.

The analyzed FA and ADC cut off values were presented as mentioned in Tables 1, 2 .

\section{Fistula type correlation with inflammatory activity}

Fistula type correlation with clinical activity condition showed no relevance with specific fistula type (Fig. 6), with $P$-values ranging from 0.066 and 0.443 .

\section{Conventional MRI correlation with inflammatory activity}

Correlation between conventional MRI findings with clinical activity condition revealed statistical significance with P-value of 0.022 as well as $90 \%$ specificity with less than $35 \%$ sensitivity, which implies being good negative modality to exclude active inflammatory conditions but with weakness regarding proving them.

After adding the ADC value to the conventional MRI assessment, the sensitivity increased to $62 \%$ and specificity increased to $74.7 \%$ when adding the FA values assessment, sensitivity increased to $67.2 \%$ and specificity increased to $95 \%$, while adding both elevated the sensitivity of MRI assessment to $74.7 \%$. 


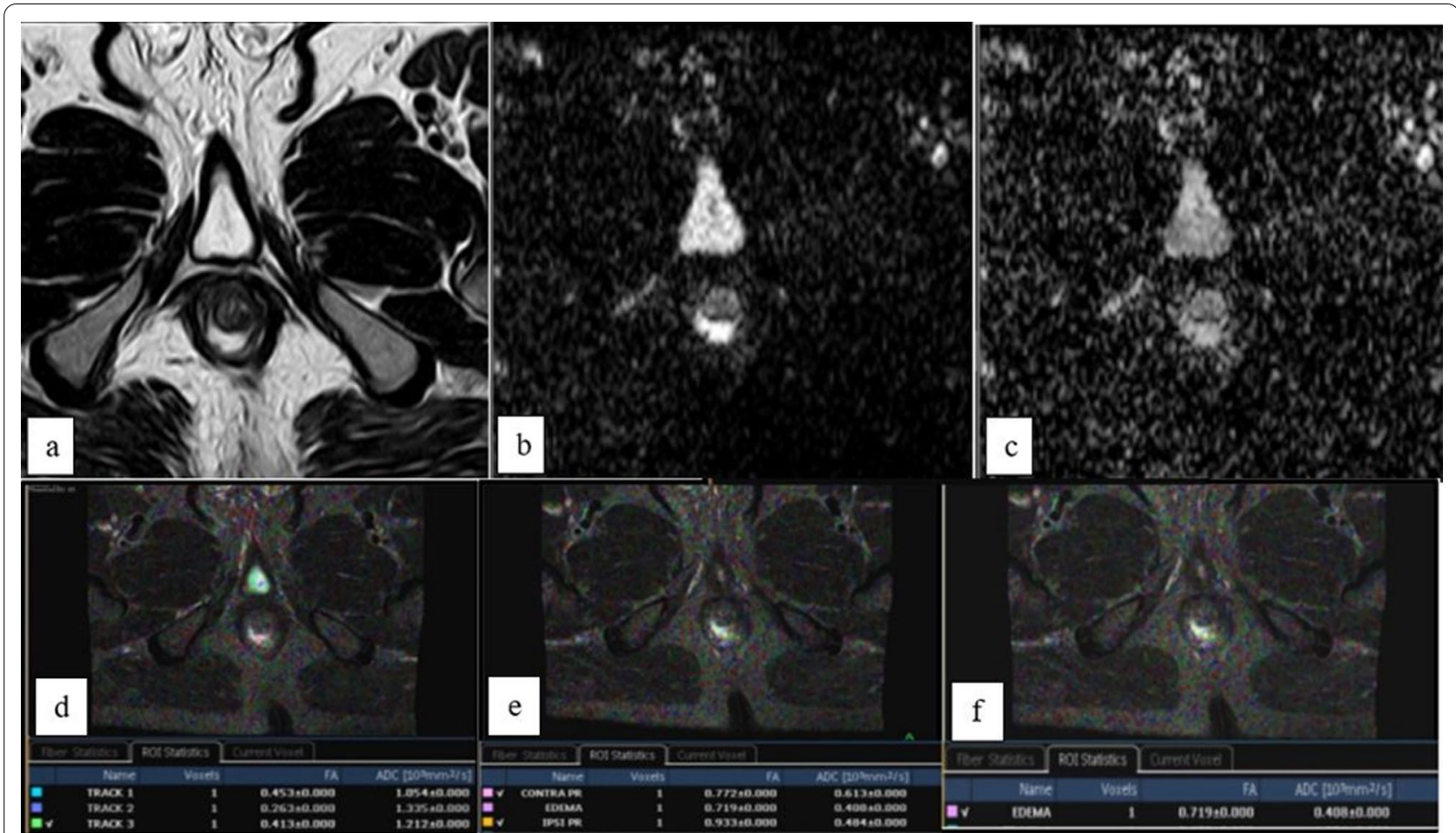

Fig. 7 a axial T2WI, b DWI and c ADC maps for an inter-sphincteric track surrounding the sphincter from 6 to 9 O'clock with internal opening breaching the internal sphincter at 7-8 O' clock and showing high T2WI SI bright DWI signal with still high ADC signal (not true restriction) in spite of being clinically and DTI active with low FA and ADC values as shown in axial DTI/T2 fused images with ROI (FA and ADC) values placed in $\mathbf{d}$ the inter-sphincteric track, e ipsilateral and contralateral sphincters and $\mathbf{f}$ surrounding edema area with FA values $0.413,0.933$, and 0.772 and 0.719 with ADC values $1.212,0.484,0.613$ and $0.408 \times 10^{3} \mathrm{~mm}^{2} / \mathrm{s}$, respectively

\section{DWI/ADC map qualitative correlation with inflammatory activity}

Yet, correlation of the diffusion weighted images and ADC maps (qualitative) findings with the clinical condition showed no statistical significance regarding the activity, with most of the tracks (54 cases) showed bright signal on DWI, yet with a large proportion (39 cases) of them showing also bright ADC map appearance (not true diffusion restriction) (Figs. 7, 8).

\section{Discussion}

The recent introduction of diffusion tensor imaging (DTI) in assessment of fistulae activity condition has been growing recently and showing promising results. By the means of quantitative ADC and FA assessment, DTI sequences helped in differentiating inflammatory conditions depending on the different directions and behavior of the disturbed normal Brownian movement of the water molecules.

In this study quantitative DTI accuracy in inflammatory condition assessment when compared to clinical/ surgical data, conventional MRI assessment as well as diffusion weighted imaging, it revealed much higher accuracy in the inflammatory activity detection with the FA and ADC reaching $100 \%$ and $89.7 \%$ sensitivity for the track area.

These results were found consistent with Wang et al. [5] who found that FA values are relatively lower in the PIA cases than in NIA and the differences were statistically significant with Pvalues (FA) 0.009, 0.004 values for the track and edema areas in Yu Wang study compared to $P$-values 0.000 and 0.000 in our study. While for the $A D C$ values in the fistula area in our study revealed higher values in PIA compared to lower values in Wang et al. [5] with still statistically significant differences with the Pvalue (ADC) 0.004 in Wang et al. study compared to $P$ value 0.000 in our study [5]. In addition, our study also concluded higher statistically significant ADC values in the edema area, ipsilateral sphincter as well as the contralateral sphincter with $P$ values $0.000,0.001$ and 0.007 , respectively.

Our study also assessed the role of conventional MRI in detecting the inflammatory activity and was found to be of high specificity (90\%) but lacking good sensitivity (34.4\%) and so being good negative only. This was much 


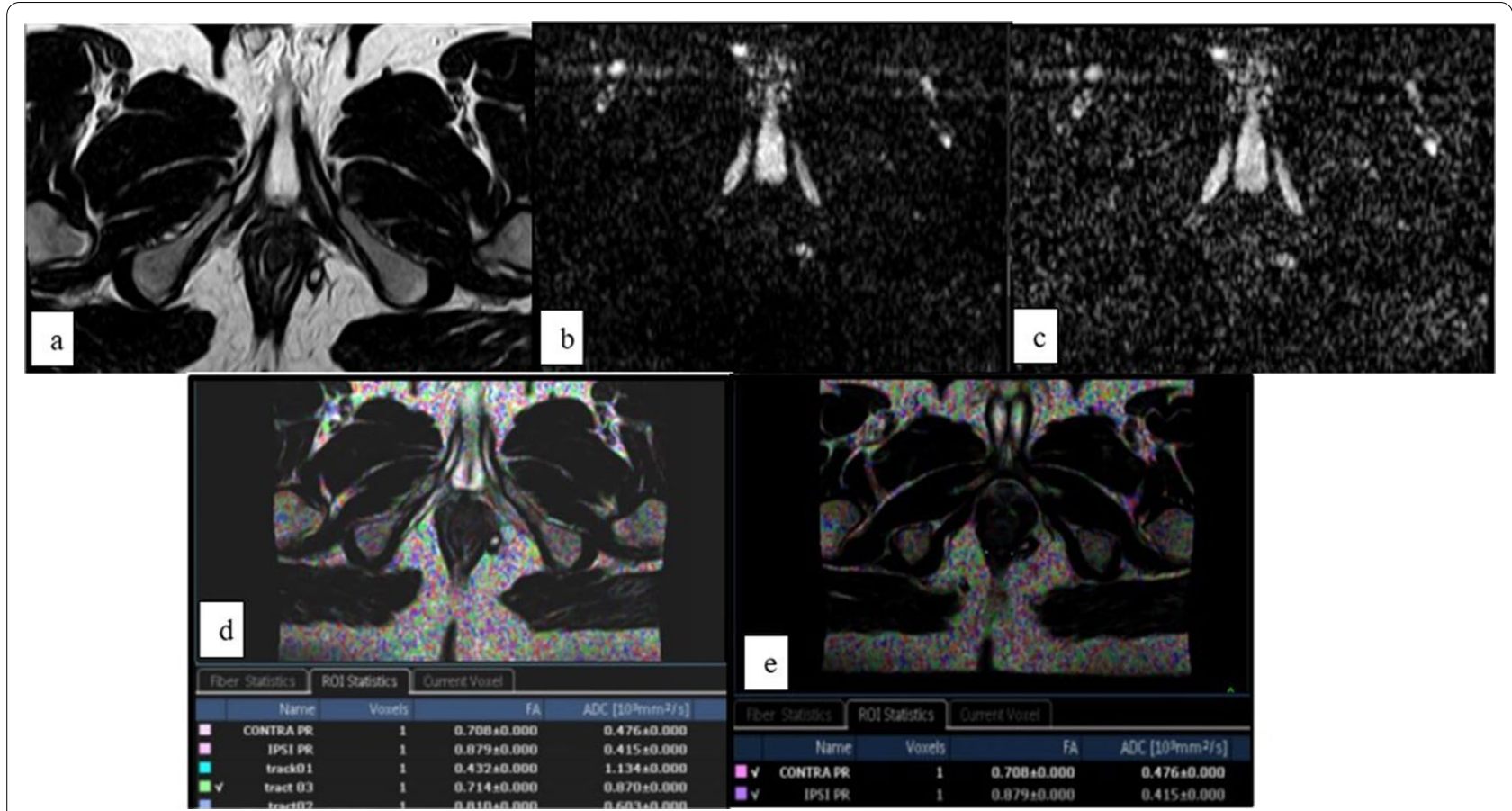

Fig. 8 a axial T2WI, b DWI and c ADC maps for a trans-sphincteric track breaching the sphincter at 3 O' clock showing bright T2, DWI WI and ADC map signal indicating absence of true diffusion restriction with being clinically inactive with high FA and ADC values as shown in axial DTI/T2 fused images with $\mathrm{ROI}$ (FA and $\mathrm{ADC}$ ) values placed in the trans-sphincteric track (d), ipsilateral and contralateral sphincters e with FA values $0.714,0.879$, and 0.708 and $A D C$ values $0.870,0.415$ and $0.476 \times 103 \mathrm{~mm}^{2} / \mathrm{s}$, respectively

improved when adding the DTI ADC value to the assessment it increased to $62 \%$ and when adding the DTI FA values assessment, it increased to $67.2 \%$, while adding both elevated the sensitivity of MRI assessment to $74.7 \%$. While the specificity was $79.25 \%$ when adding DTI (ADC) and increased to 95\% when adding DTI (FA).

These results agreed with the results reported by Algazzar et al. who studied Preoperative MRI of perianal fistula evaluation and its impact on surgical outcome and found out that MRI is valuable and accurate for preoperative investigation for perianal fistula evaluation. They also found that preoperative MRI could predict the severity of perianal disease with sensitivity, specificity, and accuracy rates of $75 \%, 92 \%$, and $84.6 \%$, respectively. Diffusionweighted magnetic resonance imaging (DWI) provided high sensitivity and accuracy with $100 \%$ specificity for fistula visualization and highest sensitivity, specificity, and accuracy for detection of cavities $>3 \mathrm{~mm}$ in diameter. They also found that DWI provided the highest specificity rate on ROC curve analysis among the three MRI pulse sequences (DWI, STIR, and T1 post-contrast). And so, they concluded that DW-MRI is a valuable sequence in diagnosing fistulas yet is more informative when added to other sequences as STIR in their study and FA and $A D C$ values in ours, to be effective in detecting fistulas activity [6].
We also found that qualitative assessment of the different signals of the tracks in DWI and ADC maps in positive and negative inflammatory activity groups was found statistically insignificant ( $P$ value 0.263 and 1.0$)$ in detecting perianal fistula activity, being indifferent in both conditions agreeing with Mohsen et al. [7], who concluded that perianal fistulas without abscesses had variable visibility scores and it was not significantly different between positive and negative inflammatory groups with $P$ value 0.45 . They concluded that the ADC values for perianal fistulas was $1.39 \pm 0.4 \times 10^{-3} \mathrm{~mm}^{2} / \mathrm{s}$, while for abscesses, it was $0.8 \pm 0.66 \times 100^{-3} \mathrm{~mm}^{2} / \mathrm{s}$, with significant difference between both entities $(P=0.001)$. There was no significant difference between PIA and NIA groups regarding the $\mathrm{ADC}$ value of perianal fistulas (PIA $1.3 \pm 0.5 \times 10^{-3} \mathrm{~mm}^{2} / \mathrm{s}$ and NIA $1.43 \pm 0.4 \times 10^{-3} \mathrm{~mm}^{2} / \mathrm{s}$, $P=0.45)[7]$.

They also concluded that DWI had a good performance in the evaluation of perianal inflammatory disease. However, combined DWI and T2W evaluation had better performance which was not significantly different from combined $\mathrm{T} 2 \mathrm{~W}$ and post-contrast images [7].

These results were also in concordance with Baik et al. [8], who concluded that DWI (qualitative not quantitative) had comparable diagnostic performance 
to T2 WI with no special added value ( $P$ value 0.0039$)$ except for one reader out of 28 .

\section{Conclusions}

This study results revealed that FA are relatively lower in positive inflammatory activity lesions than in negative inflammatory activity lesions while the ADC values were relatively higher in positive inflammatory activity lesions than in negative inflammatory activity lesions and the differences were statistically significant having a fundamental role in the assessment of perianal fistula activity especially at the track area being of highest sensitivity and specificity. Unlike conventional MRI sequences which revealed only high specificity being a good negative modality.

\section{Study limitations}

We admit that our study had many limitations, such as relatively small sample size. Second the study included more male patients with that sex ratio imbalance could have affected the results, which also represented that the incidence of the perianal fistulas is higher in males than in females. Third, in order to insure not to lengthen the examination time we chose multiple b-values for some of the cases not all of them, but in the future, we recommend acquiring more b-values for all patients to obtain more accurate results.

\begin{abstract}
Abbreviations
ADC: Apparent diffusion coefficient; Cl: Confidence interval; DTI: Diffusion tensor imaging; DWl: Diffusion weighted images; FA: Fractional anisotropy; MRI: Magnetic resonance imaging; NPV: Negative predictive value; PPV: Positive predictive value; TSE: Turbo spin echo.
\end{abstract}

\section{Acknowledgements}

All acknowledgment goes to Cairo for facilitating this study.

\begin{abstract}
Authors' contributions
RE collected the patients' data, revised their imaging process, done postprocessing application and written the core material. FS helped the post imaging processing of imaging and data interpretation. MH reviewed the net results and all data work. ANM supplied the surgical information sufficient for data classification. HA reviewed results, revised the collected data and guided their statistical analysis. All authors read and approved the final manuscript.
\end{abstract}

\section{Funding}

The current study was totally conducted at Cairo university hospitals' radiology department MRI machine and workstation. No funding received.

\section{Availability of data and materials}

The datasets used and/or analysed during the current study are available from the corresponding author on reasonable request.

\section{Declarations}

Ethics approval and consent to participate

This study was approved by the Research Ethical Committee (REC) of faculty of medicine, Cairo university. Approval reference number code: D-22-2019.
Consent for publication

Not applicable.

\section{Competing interests}

The authors declare that they have no competing interests.

Received: 6 September 2021 Accepted: 13 February 2022

Published online: 25 February 2022

\section{References}

1. Halligan S et al (2006) Imaging of fistula in ano. Radiology 239:18-33

2. Matalon SA, Mamon HJ, Fuchs CS et al (2015) Anorectal cancer: critical anatomic and staging distinctions that affect use of radiation therapy. Radiographics 35:2090-2107

3. Surabhi VR, Menias CO, Amer AM et al (2016) Tumors and tumorlike conditions of the anal canal and perianal region: MR imaging findings. Radiographics 36:1339-1353

4. Van Outryve MJ, Pelckmans PA, Michielsen PP, Van Maercke YM (1991) Value of transrectal ultrasonography in Crohn's disease. Gastroenterology 101(5):1171-1177

5. Wang Y, Gu C, Huo Y, Han W, Yu J, Ding C, Zhao X, Meng Y, Li C (2018) Diffusion tensor imaging for evaluating perianal fistula: feasibility study. Medicine (Baltimore) 97(29):e11570

6. Algazzar H, Eldib D, Bahram M, Zaher N (2019) Preoperative MRI of perianal fistula evaluation and its impact on surgical outcome. Egypt J Radiol Nucl Med 50:71

7. Mohsen L, Osman N (2020) Diffusion-weighted imaging in the evaluation of perianal fistula and abscess. Egypt J Radiol Nucl Med 51:71

8. Baik J, Kim SH, Lee Y, Yoon JH (2017) Comparison of T2-weighted imaging, diffusion-weighted imaging and contrast-enhanced T1-weighted MR imaging for evaluating perianal fistulas. Clin Imaging 44:16-21

\section{Publisher's Note}

Springer Nature remains neutral with regard to jurisdictional claims in published maps and institutional affiliations.

\section{Submit your manuscript to a SpringerOpen ${ }^{\odot}$ journal and benefit from:}

- Convenient online submission

- Rigorous peer review

- Open access: articles freely available online

- High visibility within the field

- Retaining the copyright to your article

Submit your next manuscript at $\boldsymbol{\nabla}$ springeropen.com 